\title{
SYMMETRIC DIFFERENTIAL EXPRESSIONS
}

\author{
HENRY J. RIBLET
}

1. Introduction. In a recent article, ${ }^{1}$ it is shown that if

$$
f\left(x_{1}, \cdots, x_{n}\right)=\sum c_{\nu_{01}}, \ldots, \nu_{k n} x_{1}^{\nu_{10}} \cdots\left(x_{1}^{(1)}\right)^{\nu_{11}} \cdots\left(x_{n}^{(k)}\right)^{\nu_{k n}},
$$

where $x_{i}^{(k)}$ represents the $k$ th derivative of $x_{i}$ and $f\left(x_{1}, \cdots, x_{n}\right)$ is unchanged by all permutations of the variables, then

$$
f\left(x_{1}, \cdots, x_{n}\right)=\frac{1}{D^{p}} \sum C_{\lambda_{01}}, \cdots, \lambda_{k n} a_{1}^{\lambda_{10}} \cdots\left(a_{1}^{(1)}\right)^{\lambda_{11}} \cdots\left(a_{n}^{(k)}\right)^{\lambda_{k n}}
$$

where $D$ is the discriminant of $x_{1}, \cdots, x_{n}$, and $a_{1}, \cdots, a_{n}$ are the elementary symmetric functions or E.S.F.'s. In applications to problems involving the differential equations satisfied by algebraic functions or the algebraic properties of the solutions of algebraic differential equations, it is desirable to have some method for passing from (1) to (2). The proof of the basic theorem, although constructive, gives a method that is prohibitively laborious and will of ten introduce unnecessary powers of $D$. It is the object of this paper to present an exhaustion procedure which greatly simplifies the work and obviates the latter danger.

2. Preliminaries. It is no restriction to limit ourselves to symmetric differential functions generated by a single term of (1). Term $A$ of such a function will be said to be of higher order than term $B$ if the first exponent $\nu_{i j}$ in $A$ which differs from the corresponding exponent $\nu_{i j}^{\prime}$ in $B$ is the larger.

THEOREM 1. The exponents of any power product of the derivatives of the E.S.F.'s are uniquely determined by the highest order term; and the exponents $\nu_{i j}$ of the highest order term satisfy the inequalities, where $j=1, \cdots, n-1$,

$$
\nu_{0 j}-\nu_{0 j+1} \geqq \sum_{i=1}^{k} \nu_{i j+1}
$$

PRoof. It is clear that the highest order term in $a_{i}^{(j)}$ is $x_{1} \cdots x_{i-1} x_{i}^{(j)}$ and that the highest order term in

Presented to the Society, October 28, 1939 under the title Symmetric differential expressions and applications; received by the editors January 31, 1942.

${ }^{1} \mathrm{H}$. J. Riblet, Algebraic differential fields, American Journal of Mathematics, vol. 63 (1941), p. 341. 


$$
a_{1}^{\lambda_{01}} \cdots a_{n}^{\lambda_{0 n}}\left(a_{1}^{(1)}\right)^{\lambda_{11}} \cdots\left(a_{n}^{(k)}\right)^{\lambda_{k n}}
$$

is

$$
x_{1}^{\nu_{01}} \cdots x_{n}^{\nu_{0 n}}\left(x_{1}^{(1)}\right)^{\nu_{11}} \cdots\left(x_{n}^{(k)}\right)^{\nu_{k n}}
$$

where we have $\nu_{i j}=\lambda_{i j}$ for $i$ greater than zero and otherwise

Now

$$
\nu_{0 l}=\lambda_{0 l}+\sum_{i, j>l} \lambda_{i j}
$$

$$
\begin{aligned}
\nu_{0 l}-\nu_{0 l+1} & =\lambda_{0 l}+\sum_{i, j>l} \lambda_{i j}-\left(\lambda_{0 l+1}+\sum_{i, j>l+1} \lambda_{i j}\right) \\
& =\lambda_{0 l}+\sum_{i=1}^{k} \lambda_{i l+1}=\lambda_{0 l}+\sum_{i=1}^{k} \nu_{i l+1}
\end{aligned}
$$

and since $\lambda_{0 l} \geqq 0$ condition ( 3 ) is certainly satisfied. Moreover, if it is satisfied, the equations $\nu_{i j}=\lambda_{i j}$, for $i$ greater than one and (4) otherwise determine all the $\lambda$ 's uniquely.

3. The procedure. We now consider the possibility of finding expression (2) by subtracting from $f\left(x_{1}, \cdots, x_{n}\right)$ a suitable multiple of that power product of the derivatives of the E.S.F.'s which its highest order term defines, by Theorem 1 ; and then proceeding in a similar manner with the resulting function.

ThEOREM 2. A necessary and sufficient condition that (1) be expressible as a rational integral function of the E.S.F.'s and their derivatives is that the highest order term of each function obtained by successively eliminating highest order terms satisfy condition (3).

Proof. From Theorem 1 it follows that condition (3) is certainly necessary. Sufficiency also follows from this theorem if we may be assured that there are only a finite number of terms lower than the original highest order term. This follows from the fact that in each term which occurs $\sum i \nu_{i j}=\sum i \lambda_{i j}=M$, a fixed quantity.

If the conditions of Theorem 2 are not satisfied, we will encounter a symmetric differential function $f_{2}$ whose highest order term does not satisfy condition (3). The highest order term of $D$ is $x_{1}^{2 n-2} x_{2}^{2 n-4} \cdots x_{n}^{2}$. It is clear that multiplication of $f_{2}$ by a sufficiently high power $p_{2}$ of $D$ will give a function whose highest order term satisfies condition (3). Thus we can continue as before hoping that the process will terminate.

THEOREM 3. The exhaustion procedure terminates and when it does it gives the minimum power of $D$ for which an expression of the form of (2) exists. 
Proof. Let $p$ be the smallest power of $D$ for which an expression of the form (2) exists, and suppose that in the exhaustion process we have reached a point so that

$$
f\left(x_{1}, \cdots, x_{n}\right)=f_{1}+\frac{f_{2}}{D^{p_{2}}}+\frac{f_{3}}{D^{p_{3}}}+\cdots+\frac{f_{r}+f_{r}^{\prime}}{D^{p_{r}}},
$$

where $f_{i}$ denotes a rational integral function of the E.S.F.'s and their derivatives, $p_{r} \geqq p$, and $b_{r}$, the highest order term of $f_{r}^{\prime}$, does not satisfy condition (3). If we replace $f\left(x_{1}, \cdots, x_{n}\right)$ by its equivalent as given by equation (2) and multiply both sides of the result by $D^{p_{r}}$, we obtain an expression for $f_{r}^{\prime}$ as a rational, integral function of the E.S.F.'s and their derivatives. This however contradicts Theorem 1 unless $b_{r}=0$ and this implies that $f_{r}^{\prime} \equiv 0$ which in turn proves the first assertion. Now if $p_{r}>p$, we can immediately prove that $D$ divides $f_{r}$ considered as a function of the E.S.F.'s and their derivatives. This completes the proof of the theorem.

As a corollary of Theorem 2 and as an example of the fact that the power of $D$ occurring in the denominator of (2) may be less, for an extensive class of functions, than might be expected from the original construction, we prove this theorem:

THEOREM 4. An integral rational symmetric differential expression which is linear in its differentiated terms may be expressed as an integral rational function of the E.S.F.'s and their first derivatives.

Proof. It is clear that the theorem is proved, if we can show its truth for the symmetric function generated by the term

$$
\sum_{i=1}^{n} x_{i}^{a_{i}} x_{j}^{(1)}
$$

Now the highest order term for any such differential expression satisfies condition (3), for, if not, $a_{j-1} \geqq a_{j}$ and simply by interchanging $x_{j}$ and $x_{j-1}$ we could obtain a term of higher order. Thus our result follows directly from Theorem 2 .

Hofstra College 